\title{
Análise de Desempenho de Algoritmos de Roteamento e Alocação de Comprimentos de Onda em Redes WDM
}

\author{
Simone Cintra Chagas, Eber Huanca Cayo, Jacir Luiz Bordim
}

\begin{abstract}
Resumo-O desempenho de redes WDM é usualmente analisado através da probabilidade de bloqueio de uma dada requisição. É argumentado que rotas alternativas podem ter um impacto significativo na probabilidade de bloqueio de uma rede $W D M$. Neste trabalho nós analisamos o impacto de rotas alternativas em topologias em anel e em malha. Resultados mostram que, em contradição às nossas expectativas, ter rotas alternativas pode ter um impacto negativo em certos casos.
\end{abstract}

Palavras-Chave-WDM, requisições, probabilidade de bloqueio, comprimentos de onda, fixo, fixo-alternado, First-Fit,

Abstract-The performance of WDM networks are usually analized through the blocking probability of a given request. It is argued that alternative routes can have a significant impact on the blocking probability of a WDM network. In this work, we analize the impact of alternative routes in mesh and ring topologies. Results show that, contrary to our expectation, having alternative routes can have a negative impact in certain cases.

Keywords-WDM, request, wavelength, blocking probability, wavelenghts, fixed, fixed-alternate, First-Fit

\section{InTROdUÇÃO}

Em redes ópticas $W D M$, as conexões entre dois nós são estabelecidas através de canais de comprimentos de onda que viajam em um caminho completamente óptico,ou seja, um caminho sem conversão eletro-óptica na origem e sem conversão óptica-eletro no destino. Esse caminho totalmente óptico é conhecido como caminho-de-luz.

Hoje em dia, existe uma demanda crescente por redes capazes de transmitir um grande volume de dados a altas velocidades. Uma rede com centenas de nós, incrementada com a tecnologia WDM será capaz de atender essa demanda. Não obstante, para que tal tarefa seja possível, é necessário acoplar a tecnologia WDM com meios eficientes de se estabalecer rotas através das quais os dados irão fluir. O problema de encontrar um caminho, isto é, uma rota, de um nó origem até um nó destino em uma rede WDM com comprimentos de onda livres e contínuos ao longo de cada enlace do caminho não é trivial. Por isso, o problema acima é conhecido como problema RWA (Routing and Wavelength Assignment) e foi provado em [2] que o mesmo é NP-Completo. Com o objetivo de melhor usar a tecnologia WDM, um número de heurísticas vem sido propostas na literatura [4] [5] [6] [11] [14].

$\mathrm{Na}$ resolução do problema $R W A$ é usualmente assumido que o mesmo comprimento de onda $(\lambda)$ é usado ao longo de

Simone Cintra Chagas, Eber Huanca Cayo, Jacir Luiz Bordim Departamento de Engenharia Elétrica, Faculdade de Tecnologia, Universidade de Brasília, Brasília, Brasil, E-mails: simone_cintra@terra.com.br, ,hc_eber@yahoo.es,bordim@unb.br um caminho selecionado. Mais formalmente, um caminho $p$ a partir de um vértice $v$ para um vértice $v^{\prime}$ é dito ser contínuo em relação ao comprimento de onda se o mesmo $\lambda_{i}$ com $0 \leq i \leq w$ é usado através de um caminho. Essa restrição é chamada de restrição de continuidade do comprimento de onda. Sem essa restrição, um conversor óptico poderia ser usado em cada nó, no entanto tal recurso aumenta o custo da rede.

Uma maneira de tratar o problema $R W A$ é dividí-lo em dois sub-problemas: problema de Roteamento $(R)$ e problema de Atribuição de Comprimentos de Onda $(W A)$. Dessa forma o problema $R W A$ é então resolvido da seguinte maneira: primeiro, o algoritmo de roteamento seleciona a melhor rota para os nós fonte e destino; e depois, o algoritmo de Atribuição de Comprimentos de Onda tenta obter comprimentos de onda livres e contínuos ao longo do caminho selecionado. O objetivo da combinação $R / W A$ é obter a minimização do número de comprimentos de onda e a minimização da probabilidade de bloqueio das requisições geradas.

A probabilidade de bloqueio pode ser definida como a probabilidade de uma dada requisição não ser atendida por comprimentos de onda livres em uma rota e então ser bloqueada. Formalmente teríamos, dado um conjunto de requisições $K$ formado por $\mathrm{n}$ requisições $k_{i}$ com $0 \leq i \leq n$, onde cada requisição $k_{i}$ é formada por um par fonte-destino $(f, d)$, nós temos que encontrar um caminho válido entre $(f, d)$ que possui o mesmo comprimento de onda livre $\lambda_{i}$ em cada enlace do caminho. Se essa condição não for atendida a requisição é bloqueada.

Entre as estratégias de roteamento usualmente empregadas estão roteamento fixo, roteamento fixo-alternado e roteamento adaptativo. No roteamento fixo, a mesma rota é sempre selecionada para um dado par fonte-destino. Essas rotas são usualmente computadas com os algoritmos de Dijkstra ou de Bellman-Ford [5]. No roteamento fixo-alternado, múltiplas rotas são consideradas e cada nó da rede mantém uma tabela de roteamento contendo uma lista ordenada de rotas fixas para cada nó destino. No recebimeto de uma requisição de conexão para um dado par origem/destino, um comprimento de onda contínuo é buscado na primeira rota da lista. No caso de não ser encontrado nenhum comprimento de onda livre na primeira rota, a próxima rota na lista é verificada. O processo continua até que a requisição seja atendida ou as rotas sejam exauridas [9].

No roteamento adaptativo, a rota é selecionada dinamicamente e depende do estado da rede. O estado da rede é 
determinado pelo conjunto de conexões em progresso. Os tipos de algoritmos adaptativos comumente usados são roteamento adaptativo de caminho de custo-mais-curto e roteamento adaptativo de caminho menos congestionado [8].

Todos os WA's inicialmente propostos trabalham sob a restrição de continuidade de comprimento de onda. Estudos posteriores consideraram o uso de conversores de comprimentos de onda. No entanto, devido ao custo de tais dispositivos, pode não ser possível colocar conversores de comprimentos de onda em cada nó. Para ilustrar, considere o seguinte caso. Seja $\mathrm{C}$ um conjunto de conversores e $\mathrm{V}$ um conjunto de nós na rede, com $|C| \ll|V|$. Certamente deve ser desenvolvido um mecanismo para encontrar a melhor localização para se colocar conversores, o que também é um problema NP-Completo, conforme referenciado em [9]. Heurísticas para um melhor posicionamento de conversores em redes WDM já foram propostas em [15] [17]. Neste trabalho são considerados os $W A^{\prime} s$ sem conversores de comprimentos de onda. Existe um número de $W A^{\prime} s$ propostos na literatura, os mais significativos são explicados abaixo:

- Random $(R)$ : Nesse algoritmo de alocação de comprimentos de onda, são primeiramente buscados os comprimentos de onda livres para determinar o conjunto de todos os comprimentos de onda que estão disponíveis. Dentre os comprimentos de onda disponíveis, um é escolhido randomicamente [3].

- First-Fit $(F F)$ : Todos os comprimentos de onda são numerados. Quando buscamos por comprimentos de onda livres, um comprimento de onda com o menor número é considerado antes de um comprimento de onda com um número maior. $\mathrm{O}$ primeiro comprimento de onda disponível é então selecionado [5].

- Least-Used ( $L U): L U$ seleciona o comprimento de onda que é o menos usado na rede, de tal forma a tentar balancear a carga entre todos os comprimentos de onda [11].

- Most-Used(MU): $M U$ é o oposto de $L U$ devido ao fato de tentar selecionar o comprimento de onda mais usado na rede [11].

- Least-Loaded(LL): É uma heurística designada para redes multi-fibras que seleciona o comprimento de onda que possui a maior capacidade residual no enlace mais carregado ao longo da rota $p$ [11].

- Max-Sum $\left(M \sum\right)$ :Também é proposto para redes multifibras mas pode também ser aplicado para o caso de uma única fibra. O MAX-SUM(M $\sum$ ) considera todos os caminhos possíveis (caminhos de luz com suas rotas préselecionadas) na rede e tenta maximizar as capacidades dos caminhos restantes depois do estabelecimento de um caminho-de-luz [6].

- Relative Capacity Loss(RCL): É baseado no MAX-SUM e é uma tentativa de melhorá-lo. O RCL calcula a Perda de Capacidade Relativa para cada caminho em cada comprimento de onda e depois escolhe o comprimento de onda que minimiza a soma da perda de capacidade relativa em todos os caminhos [8].

- Distributed Relative Capacity Loss(DRCL): O DRCL foi proposto para ser aplicado em ambientes distribuídos. O roteamento é fixo e é computado pelo algoritmo de Bellman-Ford onde cada nó troca tabelas de roteamento com seus nós vizinhos e atualiza suas tabelas de roteamento [11].

Apesar de um número de heurísticas de atribuição de comprimentos de onda $(W A)$ e soluções de roteamento $(R)$ existirem, ainda não é claro como o desempenho, em termos de probabilidade de bloqueio, é avaliado quando existe um aumento no número de comprimentos de onda ou quando existem rotas alternativas (rotas backup). Este trabalho é uma tentativa em fornecer insights nessa direção. Mais especificamente, neste trabalho nós podemos verificar como rotas backup e a disponibilidade de comprimentos de onda adicionais impactam a probabilidade de bloqueio. Neste trabalho são consideradas duas topologias bem conhecidas, anel e malha. Os resultados mostram que o roteamento fixoalternado/First-Fit em combinação com o aumento do número de comprimentos de onda oferece um melhor desempenho que o roteamento fixo/First-Fit na topologia em malha.

O restante desse trabalho é organizado da seguinte maneira. A Seção II cita os Trabalhos Selecionados. A Seção III explica os conceitos básicos de redes WDM. Na seção IV estão os Resultados Experimentais das simulações realizadas juntamente com a probabilidade de bloqueio calculada de forma estatística. Na seção $\mathrm{V}$ está o Modelo Analítico. Na Seção VI estão as Conclusões.

\section{TRABALhos RELACIONADOS}

Aqui nós revisamos alguns resultados e contribuições que são relacionados a este trabalho. Foi mostrado em [3] que as heurísticas Random e First-Fit atinjem resultados razoáveis em uma configuração que seja formada somente por uma fibra. A vantagem do First-Fit consiste no fato de combinar baixos custos de comunicação e computação, já que não é necessário ter conhecimento global da rede [19]. A atribuição de comprimentos de onda para redes multi-fibras foi proposta em [8], onde o algoritmo $R C L$ foi proposto. Este algoritmo avalia os benefícios da adição de uma rota alternativa (rota backup) através do algoritmo fixo-alternado. É demonstrado que a adição de somente uma rota alternativa diminui consideravelmente a probabilidade de bloqueio. As topologias analisadas são NFSNET e mesh-torus $4 \times 4$.

Em [10] e em [22] foi verificado os benefícios do aumento do número de comprimentos de onda em comparação com a adição de conversores de comprimentos de onda na topologia em anel. Em [10] a análise foi feita usando o roteamento fixo com distintos $W A^{\prime} s$ tais como Random, First-Fit e $M A X$ $S U M$. Os resultados mostraram que, sob certas condições, os benefícios obtidos adicionando-se mais comprimentos de onda é quase o mesmo que o obtido com a adição de conversores.

Em [21] foi feita uma comparação entre os algoritmos Random e First-Fit em relação à probabilidade de bloqueio na topologia em anel. Nos testes realizados, o número de nós e comprimentos de onda são mantidos constantes. A probabilidade de bloqueio é calculada usando a fórmula Erlang-B. Na análise foi verificado que o mesmo cenário sem conversores 
de comprimentos de onda oferece um desempenho melhor que o algoritmo First-Fit.

Em [17], e também em [18], os autores propuseram um método para computar a probabilidade de bloqueio no momento de servir uma dada requisição. Com essa informação em mãos, é possível verificar qual o melhor $R / W A$ a ser utilizado, ou seja, aquele que possui a menor probabilidade de bloqueio. Os benefícios da proposta foram verificados por testes empíricos com topologias lineares e em anel.

\section{REDES WDM}

Existem dois mecanismos básicos de multiplexação usados em redes ópticas, Multiplexação por Comprimentos de Onda (WDM- Wavelength Division Multiplexing ) e Multiplexação Óptica por Divisão de Tempo (OTDM-Optical Time Division Multiplexing). A primeira pode ser vista como uma maneira de multiplexar vários comprimentos de onda em uma única fibra, enquanto OTDM consiste em uma técnica onde vários sinais ópticos são combinados, transmitidos juntos, e separados novamente baseados em diferentes tempos de chegada. Neste trabalho nós focamos em redes WDM.

A revolução na transmissão através de fibras ópticas iniciouse em 1992, dobrando a capacidade a cada seis meses e alcançando taxas de 10 Tbps em 2001. Em uma rede WDM, fluxos de laser são carregados em diferentes comprimentos de onda, os quais são usados para conexões fixas ponto-aponto. Essas conexões fixas são chamadas de caminhos-de-luz. A principal restrição em relação aos caminhos-de-luz é que diferentes caminhos-de-luz não podem compartilhar o mesmo comprimento de onda na mesma fibra óptica [3].

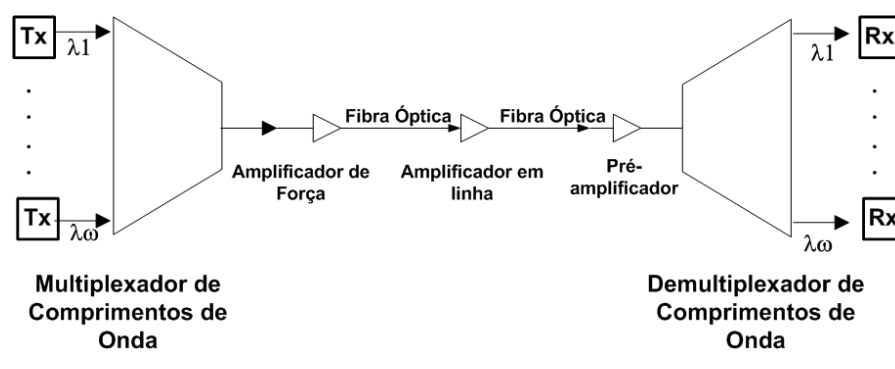

Fig. 1. Funcionamento Tecnologia WDM

Como pode ser verificado na Fig. 1, a tecnologia WDM trabalha da seguinte forma: do lado do transmissor existem $W$ transmissores independentes. Cada transmissor, denotado como $T_{x}$, é uma fonte de luz, como um laser, e é modulado independentemente como um fluxo de dados. A saída de cada transmissor é um sinal óptico, chamado comprimento de onda, denotado como $\lambda_{i}$, onde $0 \leq i \leq W$. O sinal óptico dos $W$ transmissores são combinados em um único sinal óptico pelo multiplexador e transmitidos pela fibra óptica. Do outro lado, os sinais ópticos são demultiplexados em $W$ sinais individuais, que são depois endereçados para o receptor apropriado. A amplificação é usada depois da multiplexação do comprimento de onda e antes da demultiplexação do comprimento de onda [12].

Um sistema $W D M$ ponto-a-ponto provê $W$ canais independentes, todos eles na mesma fibra. Conforme a tecnologia
WDM vai evoluindo, o número de comprimentos de onda que podem ser transmitidos na mesma fibra vai aumentando. Em outras palavras, o enlace de uma fibra pode ser aumentado sem a necessidade de adicionar-se novas fibras, o que é mais barato e consome menos tempo. A adição ou substituição de novas fibras é consideravelmente mais cara que a melhoria dos componentes necessários.

\section{Modelo DE ANÁlise}

Essa seção detalha o modelo usado para avaliar a probabilidade de bloqueio em uma rede WDM. Em particular, nós consideramos duas topologias bem conhecidas, anel e malha. Para o propósito de análise, nós consideramos um modelo tendo as seguintes características:

- As requisições são randomicamente/incrementalmente geradas e são servidas em uma maneiro FIFO;

- Requisições que não são servidas são consideradas bloqueadas;

- A Probabilidade de Bloqueio é computada na mesma forma estatística que em [21]: $P_{b}=R_{n s} / R_{g}$, onde $R_{n s}$ representa o número de requisições não servidas e $R_{g}$ representa o número de requisições geradas ;

- Todos os enlaces são bidirecionais;

- Uma vez que uma requisição é servida, permenecerá alocada durante toda a simulação;

- Os algoritmos de roteamento a serem analisados serão fixo e fixo-alternado;

- A heurística de alocação de comprimentos de onda usada ( $W A$ ) nas simulações é First-Fit.

Os resultados das simulações são calculados da seguinte maneira. Inicialmente, um pool de $K$ requisições são geradas. Cada requisição individual é denotada por $k_{i}$, onde $1 \leq i \leq K$, e é associada a um par fonte e destino $(f, d)$. A Tabela I mostra os parâmetros da simulação: topologia, estratégia de roteamento, número de comprimentos de onda $(W)$ e número de simulações $(K)$. Então, a execução de uma simulação consiste dos seguintes quatro parâmetros (topologia, estratégia de roteamento, $W, K$ ). Aqui nós usamos $K=100$ e os resultados foram obtidos a partir de uma média de cem execuções, então o número de requisições simuladas foram 10.000 no total.

TABELA I

CEnÁRIos a SEREM SIMUlados COM O ALGORITMO DE ALOCAÇão First-Fit

\begin{tabular}{|c|c|c|}
\hline topologia & roteamento & $W$ \\
\hline Anel 10 nós & fixo & {$[4,5,6,7,8]$} \\
\hline Anel 10 nós & fixo-alternado & {$[4,5,6,7,8]$} \\
\hline Malha 4x4 (16 nós) & fixo & {$[4,5,6,7,8]$} \\
\hline Malha 4x4 (16 nós) & fixo-alternado & {$[4,5,6,7,8]$} \\
\hline
\end{tabular}

\section{RESULTADOS EXPERIMENTAIS}

As topologias simuladas são ilustradas na Fig. 2. O número de comprimentos de onda inicialmente usados foi $\mathrm{W}=4$, aumentando-os em $25 \%, 50 \%, 75 \%$ e $100 \%$ 


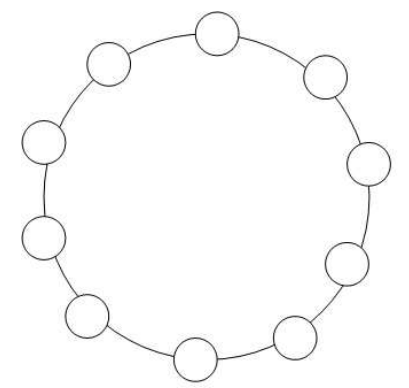

ANEL 10 NÓS

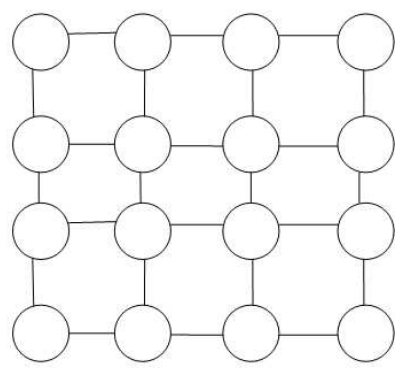

MALHA BIDIRECIONAL 4X4

Fig. 2. Topologias a serem analisadas

O algoritmo de roteamento usado na seleção do caminho mais curto é o algoritmo bastante conhecido chamado Dijkstra [11]. Na implementação do algoritmo fixo-alternado, a primeira rota é sempre a rota mais curta. Depois de computar o caminho mais curto, nós tentamos estabalecer um caminho-deluz usando a heurística First-Fit. Se nenhum comprimento de onda naquela rota está disponível, é computada uma segunda rota (disjunta em relação à anterior) e o processo é repetido. Deve ficar claro que se existem $l$ rotas disjuntas da fonte $f$ para o destino $d$, nós podemos repetir esse processo por $l$ iterações. Aqui,no entanto, foi usada somente uma rota alternativa, ou seja, o processo é repetido somente duas vezes. No caso da topologia em anel, devido ao fato de existirem somente duas rotas possíveis, a rota no sentido horário é usada como rota padrão sempre que possível.

No processo de utilização de rotas alternativas, ou seja, rotas backup, nós consideramos a eficácia de utilização de rotas alternativas, denotada por $E$. Essa medida é obtida pela divisão da soma de todas as rotas alternativas válidas $\left(\sum\right.$ Rot $\left._{\text {altvalida }}\right)$ pela soma de todas as rotas alternativas encontradas em todas as simulações. Rot altvalida representa a rota alternativa encontrada que atende a requisição gerada em uma dada execução de simulação.Rot alternativa $_{\text {representa toda rota encontrada }}$ em uma execução de simulação, independente se a requisição é satisfeita ou não pela mesma. Então, a eficácia de utilização de rotas alternativas é computada da seguinte forma:

$$
E=\left(\sum \operatorname{Rot}_{\text {altvalida }} / \sum \operatorname{Rot}_{\text {alternativa }}\right) * 100
$$

Com o intuito de ilustrar o cálculo da eficácia de utilização de rotas alternativas nós vamos considerar como exemplo uma rede em anel com 10 (dez) nós com 4 (quatro) comprimentos de onda $(\mathrm{W}=4)$, que usa o roteamento fixo-alternado/First-Fit somente com 1 (uma) rota alternativa. Depois de 100 (cem) simulações com 100 (cem) requisições os seguintes resultados são encontrados: $\sum$ Rot $_{\text {altvalida }}=500$ e $\sum$ Rot $_{\text {alternativa }}=$ 8000 , o que gera uma eficácia de utilização das rotas alternativas de $E=6,2 \%$, como pode ser verificado na Fig. 4 .

Os resultados das simulações para a topologia em anel são mostrados na Fig. 3. Como pode ser visto, o uso do roteamento fixo/First-Fit com o aumento no número de comprimentos de onda disponíveis reduz a probabilidade de bloqueio em uma

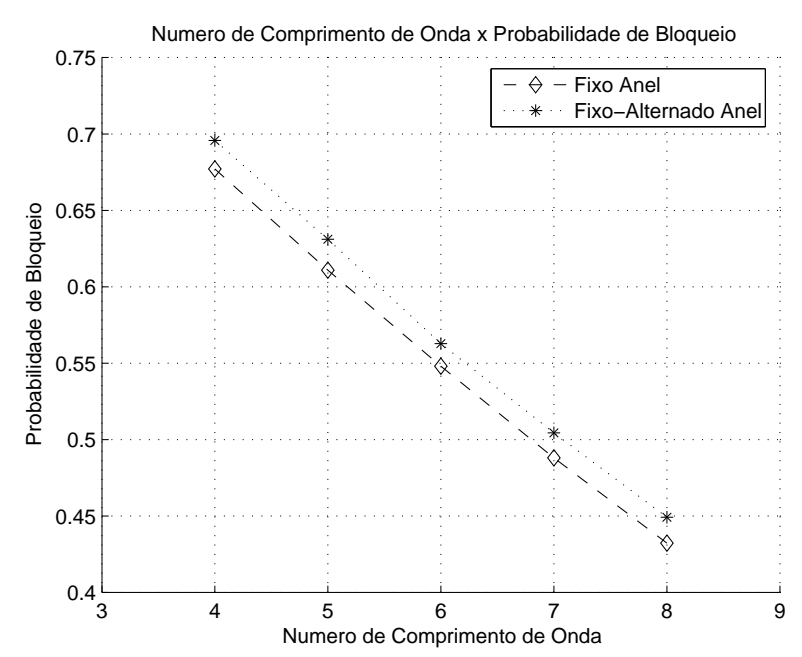

Fig. 3. Probabilidade de bloqueio na topologia em anel com roteamentos fixo/fixo-alternado

média de $0,06 \%$. O uso do roteamento fixo-alternado/FirstFit com o aumento no número de comprimentos de onda disponíveis reduz também a probabilidade de bloqueio em uma média de $0,06 \%$. Nós podemos observar através da Fig. 4 e da Tabela II que a eficácia de utilização de uma rota alternativa na topologia em anel varia de $6,1 \%$ com $W=4$ até $26 \%$ com $W=8$. A partir dessas observações nós podemos concluir que o aumento do número de comprimentos de onda oferece um melhor custo/benefício que o uso de uma única rota alternativa.

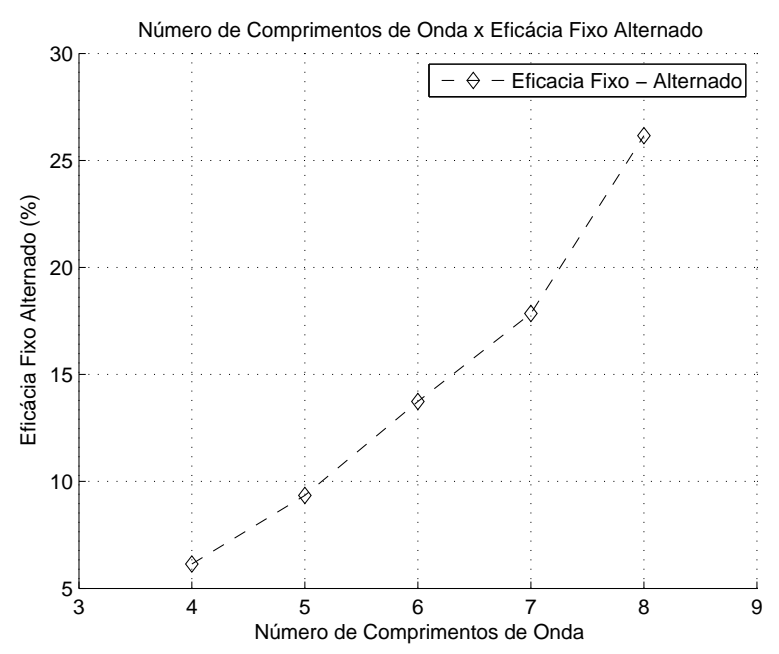

Fig. 4. Número de Comprimentos de Onda/Eficácia Roteamento fixoalternado/First-Fit na topologia em anel

Como mostrado na Fig. 5, o aumento no número de comprimentos de onda usando o roteamento fixo/First-Fit na topologia em malha diminui a probabilidade de bloqueio em aproximadamente $0,07 \%$ em média. No entanto, o roteamento fixo-alternado/First-Fit explora melhor a disponibilidade de rotas adicionais disjuntas e é capaz de servir um maior número de requisições, oferecendo uma redução considerável na probabilidade de bloqueio. 


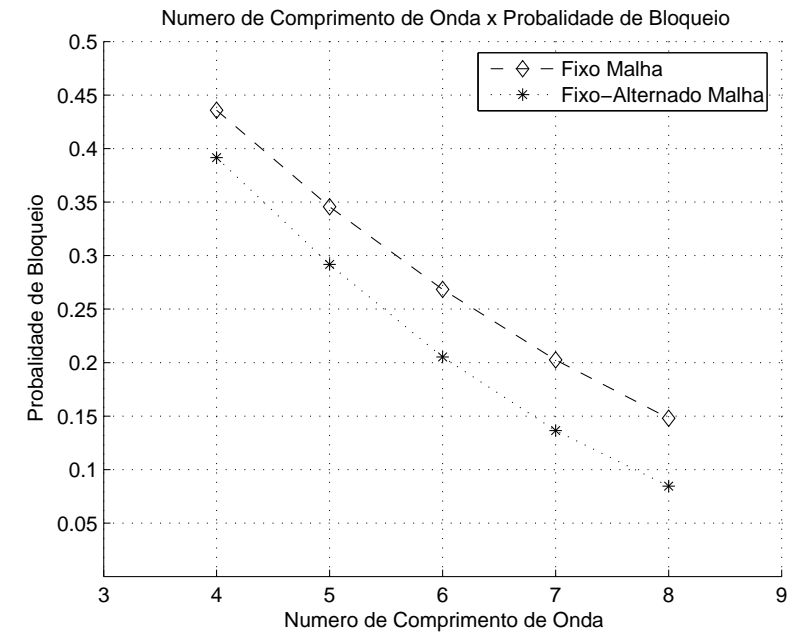

Fig. 5. Probabilidade de bloqueio na topologia em malha com roteamentos fixo/fixo-alternado

TABELA II

ANÁLISE EFicÁcia RoteAmento FiXo-Alternado/First-Fit NA TOPOLOGIA EM ANEL

\begin{tabular}{|c|c|c|c|}
\hline$W^{\prime} S$ & $\begin{array}{c}\text { Fixo } \\
\text { Atend.|Bloq. }\end{array}$ & $\begin{array}{c}\text { Alternado } \\
\text { Atend.|Bloq. }\end{array}$ & $\begin{array}{l}\text { Eficácia. } \\
\text { Alternado }\end{array}$ \\
\hline 4 & \begin{tabular}{l|l}
$53 \%$ & $47 \%$
\end{tabular} & $51 \% 149 \%$ & $6,1 \div$ \\
\hline 5 & $62 \% \quad 38 \%$ & $61 \% 139 \%$ & $9,3 \div$ \\
\hline 6 & $71 \% \quad 29 \%$ & $70 \% 130 \%$ & $13,7 \div$ \\
\hline 7 & $78 \% 122 \%$ & $79 \% 121 \%$ & $17,8 \%$ \\
\hline 8 & $85 \% \quad 15 \%$ & $87 \% \mid 13 \%$ & $26 \%$ \\
\hline
\end{tabular}

Através da Fig. 6 e da Tabela III pode ser verificado que a eficácia de utilização de rotas alternativas no roteamento fixoalternado/First-Fit na topologia em malha varia de $46 \%$ com $W=4$ até $98 \%$ com $W=8$, o que justifica o custo de utilização de uma rota alternativa com o objetivo de diminuir a probabilidade de bloqueio.

TABELA III

AnÁlise EFICÁCIA RoteAmento FIXO-Alternado/First-Fit NA TOPOLOGIA EM MALHA

\begin{tabular}{|c|c|c|c|}
\hline$W^{\prime} S$ & $\begin{array}{c}\text { Fixo } \\
\text { Atend.|Blog. }\end{array}$ & $\begin{array}{l}\text { Alternado } \\
\text { Atend.|Bloq. }\end{array}$ & $\begin{array}{l}\text { Eficácia. } \\
\text { Alternado }\end{array}$ \\
\hline 4 & \begin{tabular}{l|l}
$79 \%$ & $21 \%$
\end{tabular} & \begin{tabular}{l|l}
$87 \%$ & $13 \%$
\end{tabular} & $46 \%$ \\
\hline 5 & $88 \% 12 \%$ & $95 \% 15 \%$ & $63 \div$ \\
\hline 6 & $93 \%$ & $98 \div 12 \%$ & $78 \div$ \\
\hline 7 & $96 \%$ & $99 \% 11 \%$ & $93 \div$ \\
\hline 8 & \begin{tabular}{l|l}
$98 \%$ & $2 \%$
\end{tabular} & $99,9 \div \mid 0,1 \%$ & $98 \div$ \\
\hline
\end{tabular}

\section{CONCLUSÕES}

Através das simulações, está claro que a topologia da rede possui um impacto significativo no desempenho do $R / W A$ usado. Além do mais, o desempenho depende de um número de parâmetros que podem ser cuidadosamente analisados antes de decidir se deve-se aumentar o número de rotas alternativas ou aumentar o número de comprimentos de onda.

Neste trabalho, os resultados obtidos mostram que o roteamento fixo-alternado/First-Fit juntamente com o aumento no

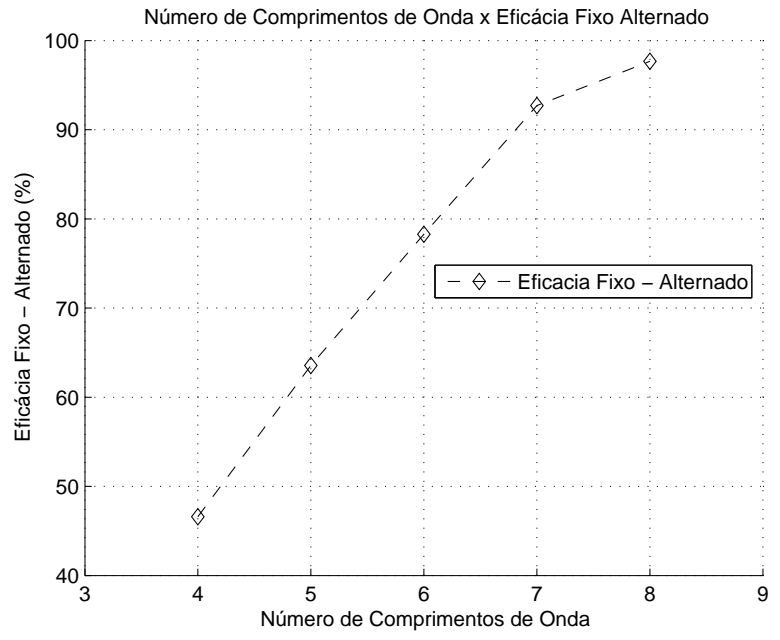

Fig. 6. Número de Comprimentos de Onda / Eficácia roteamento fixoalternado/First-Fit na topologia em malha

número de comprimentos de onda, apresenta o melhor desempenho na topologia em malha. Na topologia em anel, a adição de uma única rota alternativa oferece quase o mesmo desempenho que a adição de mais comprimentos de onda usando-se o roteamento fixo/First-Fit. Tal situação acontece porque na topologia em anel a rota alternativa será no mínimo igual ou maior que a rota principal e rotas alternativas maiores oferecem uma maior probabilidade de bloqueio.Já na topologia em malha está claro que existem um maior número de possibilidades de rotas alternativas que na topologia em anel e os caminhos são mais curtos, o que aumenta a chance de uma certa requisição ser atendida, diminuindo dessa forma a probabilidade de bloqueio.

\section{Agradecimentos}

Agradeço primeiramente a Deus por ter me dado forças pra continuar a persistir na realização deste trabalho apesar das adversidades. Agradeço também aos meus pais e colegas de Doutorado pelas palavras de incentivo nos momentos de desânimo.

\section{REFERÊNCIAS}

[1] Internet Traffic to Keep Doubling, http://www.pcworld.com/article/id,109715-page,1/article.html, last acess in April, 2008.

[2] I. Chlamtac, A. Ganz, G. Karmi, Lightpath Communications: An Approach to High Bandwidth Optical Wan's. IEEE,1992.

[3] R. Ramaswami e K. N. Sivarajan," Routing and Wavelength Assignment in All Optical Networks, IEEE ACM Transactions on Networking, v. 3, $\mathrm{n}^{\circ}$ 5, Outubro, 1995.

[4] E. Karasan, E. Ayanoglu Effects of Wavelength Routing and Selection Algorithms on Wavelength Conversion Gain in WDM Optical Networks, IEEE ,1996.

[5] S. Subramanian e A. R. Barry, Wavelength Assignment in Fixed Routing WDM Networks, IEEE,1997.

[6] R. Barry, S. Subramanian, The MAX-SUM wavelength assignment algorithm for WDM ring networks, Optical Fiber Communications 1997.

[7] M. M. Freire, A. M. F. Carvalho, Estimativa da Probabilidade de Bloqueio em Redes Ópticas WDM com topologia em anel e com encaminhamento de comprimento de onda, CRC ,1998. 
[8] X. Zhang e C. Qiao, Wavelength Assignment for Dynamic Traffic in Multi-fiber WDM Networks, 7th International Conference on Computer Communications and Networks (ICCCN'98),1998.

[9] S. Ramamurthy e B. Mukherjee, Fixed-Alternate Routing and Wavelength Conversion in Wavelength-Routed Optical Networks,IEEE/ACM Transactions on Networking ,2002.

[10] D. R. Campelo, R. Camelo, H. Waldman , Estratégias de Ampliação da Capacidade de Anéis Ópticos com Bloqueio, SBrt 2000.

[11] H. Zang, J. P. Jue, B. Mukherjee, A Review of Routing and Wavelength Assignment Approach for Wavelength Routed Optical WDM Networks, Optical Networks Magazine, Janeiro , 2000.

[12] T. H. Cormen, C. E. Leiserson , L. R. Rivest, C. Stein , Introduction to Algorithms, Second Edition, McGraw -Hill Book Company, 2001

[13] M. A. Ali, A. Shami , C. Assi , Y. Ye , Architectural Options in the Next-Generation Networks Paradigm: Is Optical Internet the Answer?,Photonic Network Communications, 3:1/2, 7-21, 2001

[14] H. Zang , J. P. Jue , L. Sahasrabuddhe , R. Ramamurthy , B. Mukherjee, Dynamic Lightpath Establishment in Wavelength-Routed WDM Networks,IEEE Communications,Setembro, 2001.

[15] J. Strand, R. Doverspike, Importance of Wavelength Conversion in an Optical Network,Optical Networks Magazine, Maio/Junho, 2001.

[16] X. Sun , Y. Li , I. Lambadaris , Y. Q. Zhao, Performance Analysis of First-Fit Wavelenght Assignment Algorithm in Optical Networks, 7th ConTEL, 2003.

[17] B. Li ,X. Chu , Routing and Wavelength Assignment vs. Wavelength Converter Placement in All- Optical Networks, IEEE ,2003.

[18] H. Waldman, D. R. Campelo, R. C. A. Júnior, Uma Nova Abordagem para Estimação de Probabilidades de Bloqueio em Redes de Roteamento de Comprimento de Onda Lineares, SBrt 2003.

[19] H. Waldman , D. R. Campelo , Analytical Calculation of Blocking Probabilities in WDM Rings with Wavelength Conversion, IEEE ,2005.

[20] D. R. Campelo , R. C. A. Junior , H. Waldman , Blocking Probabilities Generated by the First-Fit Wavelength Assignment Algorithm in WDM Rings, Novembro, III TIDIA Workshop,2006.

[21] A. Wason, R. S. Kaler, Wavelength Assignment Problem in Optical WDM Networks, IJCNS International Journal of Computer Science and Network Security,v. 7, $\mathrm{n}^{\circ}$ 4, Abril, 2007

[22] Júnior R. C. A. A., Alocação de Rota e Comprimento de Onda em Anéis WDM,Dissertação de Mestrado,FEEC-UNICAMP, 2001. 